\title{
The Controlled Manipulation of Parameters of the Quantum Antidot
}

\begin{abstract}
T. PyragienĖ
Semiconductor Physics Institute, A. Goštauto 11, 011008 Vilnius, Lithuania

The electron motion in a strong perpendicular magnetic field close to the impenetrable obstacle is considered by the semi-classical and quantum points of view. We investigated an influence of a shape of the forbidden region to the formation of the plateaux in the one electron energy spectrum and transmissions between dot and antidot states. In the semi-classical regime electrons can be treated as charged particles with well-defined trajectories pinned to the potential wall. This approach, combined with singular equation technique in quantum calculations, has given us a possibility to investigate stripe-shaped and bow-shaped antidots with sharp edges and to find a way for controlled manipulation of the parameters of systems with an effort to get desired physical properties.
\end{abstract}

PACS numbers: 73.20.Mf, 85.35.-p, 03.65.-w

\section{Introduction}

The main goal of nanotechnology is the controlled manipulation of the microstructure with an effort to get desired physical properties. In particular, it is important to know how to control such parameters of the quantum antidot (QA) as its size and shape. These parameters effect the electronic spectrum of QA and significantly influence on characteristics of devices in various applications [1].

In the semi-classical regime the electrons can be treated as charged particles with well-defined trajectories pinned to the potential wall [2]. In view of the fact that these systems have the shapes with sharp band edges the singular integral equation technique [3] was applied. This approach, combined with quantum calculations, has given us a possibility to investigate round, stripe-shaped [4] and bow-shaped antidots.

\section{Model}

We consider the electron moving in $x y$-plane which is shown in Fig. 1. The antidot, the infinitely thin impenetrable line, is indicated by the grey color. We solve the Schrödinger equation 


$$
(H-E) \Psi(\boldsymbol{r})=0
$$

with the following dimensionless Hamiltonian:

$$
H=-\frac{1}{2}[\nabla+\mathrm{i} \boldsymbol{A}(\boldsymbol{r})]^{2},
$$

where the perpendicular magnetic field is described by the vector potential in the symmetric gauge $\boldsymbol{A}(\boldsymbol{r})=(-y, x) / 2$. We use the following notation for $2 \mathrm{D}$ vectors $\boldsymbol{r}=(x, y)$. The energy is measured in $\hbar \omega_{\mathrm{c}}\left(\omega_{\mathrm{c}}=e B / m c\right)$ units, and the coordinates - in magnetic length $l_{B}=\sqrt{c \hbar / e B}$ units. The antidot is taken into account by hard wall boundary condition

$$
\Psi(\boldsymbol{r})=0 .
$$

Besides, the wave function satisfies zero boundary conditions at the infinity $\left.\Psi(\boldsymbol{r})\right|_{r \rightarrow \infty}=0$.

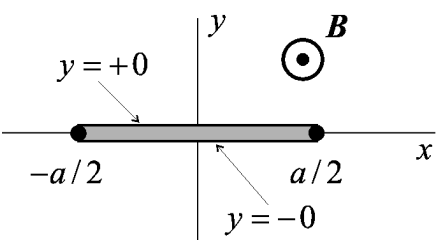

Fig. 1. Layout.

Making use of the Green theorem the above two-dimensional problem can be transformed into one-dimensional integral equation for stripe-shaped antidot. In this way the energy spectrum and the distribution of the density of charge were obtained [4]. There the semi-classical approach was used and the influence of the obstacle on the classical electron trajectories was taken into account via scattering events. The degeneracy of semi-classical levels corresponding to two circle classical trajectories was estimated. In the case of the bow-shaped antidot the same algorithm was applied and the distribution of the density of charge and the energy spectrum with lines bounded the region where the degeneracy of quasi-classical levels is equal to one were gained. This calculations is the basis for the control of characteristics of systems by changing their sizes and shapes.

\section{Results}

The energy spectrum of the bow-shaped antidot is shown in Fig. 2. Here dashed lines bound the region where the degeneracy of quasi-classical levels equals 1. In Fig. 3 the electron density for the point on the semi-classical level is shown. As we can see changing the angle of the bow gives totally different states of the system proceeding from the states with the plateau of the energy branches 
in the case of small angles of the bow and ending with transmissions between dot and antidot as its shape tends to circle. As is shown in Fig. 4, in contrast to a stripe there are transmissions between states of dot and antidot in the bow-shaped antidot.

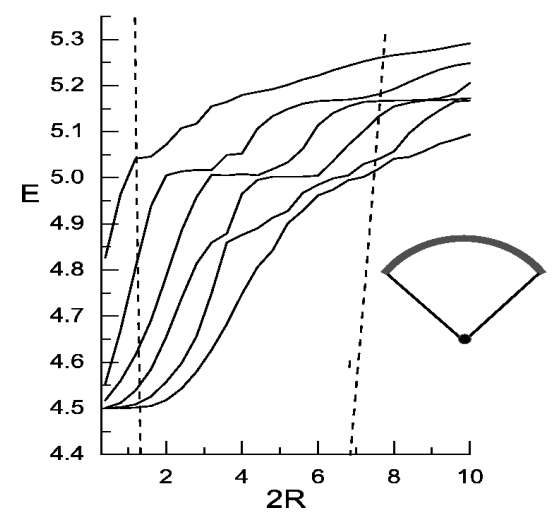

Fig. 2. The energy spectrum of the bow-shaped antidot with the angle of the bow equal to 3 .
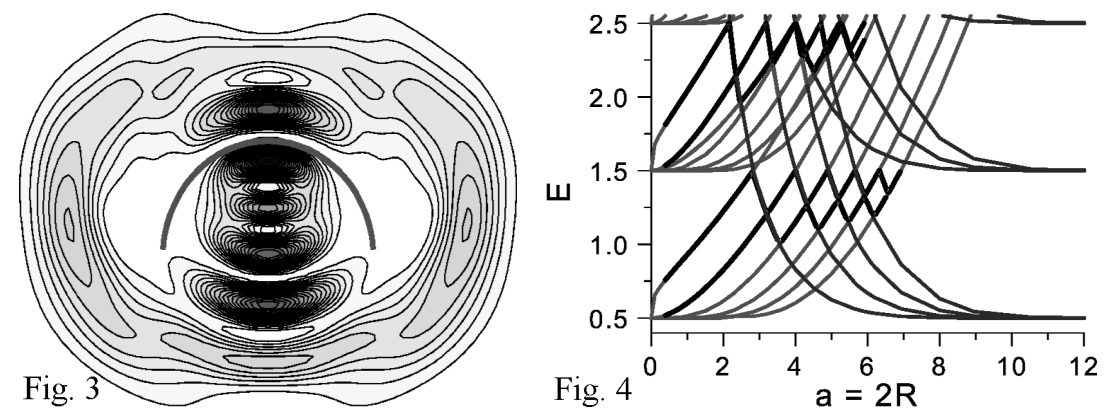

Fig. 3. The electron density for the bow with the angle equal to 3 .

Fig. 4. The energy spectrum of the bow-shaped antidot with the angle of the bow equal to 6.28 .

\section{Conclusions}

The investigation of semi-classical trajectories in addition to quantum mechanical calculations for one electron antidot gave a way for controlled manipulation of the parameters of microstructure with an effort to get desired physical properties.

In particular, one can control the length of the energy plateau in the stripeand bow-shaped antidot changing the cyclotron radius of electron trajectory, which 
is bound to its energy. In addition, the varying of the angle of the bow is a way to get the determined length of the plateau of the bow-shaped antidot. Furthermore, there are transmissions between dot and antidot states in the bow-shaped antidot with the shape tending to the round.

It is particularly remarkable that only changing the angle of the bow one can obtain the totally different states of the system proceeding from states with the plateau of the energy branches in the case of small angles of the bow and ending with transmissions between dot and antidot.

\section{References}

[1] G. Kirczenov, A.S. Sachrajada, Y. Feng, R.P. Taylor, L. Henning, J. Wang, P. Zawadski, P.T. Coleridge, Phys. Rev. Lett. 72, 2069 (1994).

[2] X. Kleber, G.M. Gusev, U. Gennser, D.K. Maude, J.C. Portal, D.I. Lubyshev, P. Basmaji, M. de P.A. Silva, J.C. Rossi, Yu.V. Nastaushev, Phys. Rev. B 54, 13859 (1996).

[3] K. Horngerger, U. Smilansky, Phys. Rep. 367, 249 (2002).

[4] A. Matulis, T. Pyragiene, Phys. Rev. B 67, 045318 (2003). 